\title{
The relationship between chiasma frequency and bivalent length: Effects of genotype and supernumerary chromosomes
}

\author{
M. I. Cano, \\ N. Henriques-Gil, \\ P. Arana and \\ J. L. Santos
}

Departamento de Genética, Facultad de Biología, Universidad Complutense, 28040 Madrid, Spain.

In the grasshopper Eyprepocnemis plorans familial and individual genotypes have significant effects either on chiasma frequency or on bivalent length; the effect of $B$ chromosomes depends on the particular family considered which derives in the absence of an overall effect of supernumeraries. This study confirms previous reports in that there is a significant positive correlation between chiasma frequency and bivalent length operating at a cellular rather than specific bivalent level. Nevertheless, the correlation between both variables is scarcely affected when the contributions of the different factors are eliminated. In addition, the effects of B chromosomes within the different families on chiasma frequencies are not parallel to the correspondent effects on bivalent length. These facts indicate that, despite such correlation, the influence of $\mathbf{B}$ chromosomes or familial and individual genotypes on chiasma frequency does not operate through changes in chromosome length at first meiotic prophase.

\section{INTRODUCTION}

Chiasma frequency is known to depend both on genetic and environmental components (Nolte et al., 1969; Shaw, 1971; 1972) including factors such as the presence of supernumerary chromosomes (for review see Jones and Rees, 1982). The adaptative significance of the observed variation in chiasma frequency remains unclear and there is some controversy on this subject (for instance Rees and Ahmad, 1962; Nolte, 1968; Shaw, 1972; Rees and Dale, 1974; Dearn, 1975; Maynard-Smith, 1977). Despite the existence of many factors affecting this parameter we lack a clear model explaining how supernumeraries or genetic and environmental factors produce variation in chiasma formation.

Henderson (1963) and Fox (1973) observed that in Schistocerca gregaria, bivalents having higher chiasma scores were longer and this was later confirmed in other materials (Couzin and Fox, 1973; Riva et al., 1984). On the basis of these findings, the structure of chromosomes is related to chiasma frequency and hence any factors affecting the former could also have an effect on chiasma frequency (Fox, 1973). Consequently Fox pro- posed this mechanism to explain the effects of different agents on chiasma formation. Riva et al. (1984) found a certain parallel between the effects of a supernumerary chromosome segment on both bivalent length and chiasma frequency in the grasshopper Euchorthippus pulvinatus gallicus; their results suggested too that genotypes also seemed to play a role in such relationship.

We have studied this problem in males of Eyprepocnemis plorans (Acrididae; Orthoptera) which show a polymorphism for supernumerary chromosomes (Henriques-Gil et al., 1982a; 1984). The individuals were developed under controlled conditions in the laboratory and so we could analyse males with 0 and $1 \mathrm{~B}$ chromosome from different families; in consequence it was possible to evaluate the contribution to the variation in chromosome length and chiasma frequency of individual and familial genotypes as well as that of B chromosomes.

\section{MATERIAL AND METHODS}

The 24 males of Eyprepocnemis plorans plorans employed in the present study correspond to part 
of the material used by Henriques-Gil et al. $(1982 b)$. Such material was grouped in families, i.e., the offspring grown under laboratory conditions from different females collected in the wild, and classified according to the number of $B$ chromosomes. Several follicles per male were analysed in C-banded preparations.

Six families are analysed here with four males in each one: two having the basic karyotype and two with one B chromosome. For each male 10 middle-diplotene cells were selected at random among those suitable for measurements-bivalents must be well and regularly squashed and identifiable (see Fox, 1973). Bivalent lengths were recorded in arbitrary units following the method described by Riva et al. (1984) using photographs of $\times 3000$ magnification.

Since C-banding does not identify all autosome pairs, the results were grouped as follows: L1 to M4, the largest chromosome pairs; M5 and M7, clearly smaller than the members of the former group; M6 and M8, very similar to the previous ones but having, in addition, a small C-band in proximal position; finally, the three smallest members of the complement can be easily distinguished, M9 is the megameric pair and S10 and S11 differ in the amount of centromeric C-heterochromatin which is much smaller in S10 than in S11.

\section{RESULTS}

\section{Sampling error}

Table 1 shows the total chromosome length and the number of chiasmata for each of the 240 cells analysed, grouped in individuals, karyotypic classes ( $0 \mathrm{~B}$ and $1 \mathrm{~B}$ chromosome) and families.

As was described in the previous section, the cells selected for measurements must accomplish some conditions. Therefore there is a possibility for sampling error if such diplotenes are not fully representative of the whole cellular population. We therefore compared the mean chiasma frequencies obtained in a wider sample, 40 diplotenes per male, from the same individuals, in which the only criterion was a clear total chiasma score, reported previously (Henriques-Gil et al., 1982b). The differences were negative in 19 of the 24 males, in three they were positive and in the remaining two males both scores were identical and in a $t$-paired analysis revealed a small significant negative deviation $(\bar{D}=-0 \cdot 193 ; t=3 \cdot 24, \mathrm{df}=23, p<0 \cdot 01)$. Mean chiasma frequencies of diplotenes suitable for bivalent measuring are usually lower than those obtained for typical cells. This can be explained if bivalents with high chiasma numbers are more easily distorted and hence inappropriate for measurements.

Table 1 Total chromosome length (arbitrary units) and number of chiasmata of all analysed cells grouped in individuals, karyotypic classes $(0$ and $1 B)$ and families

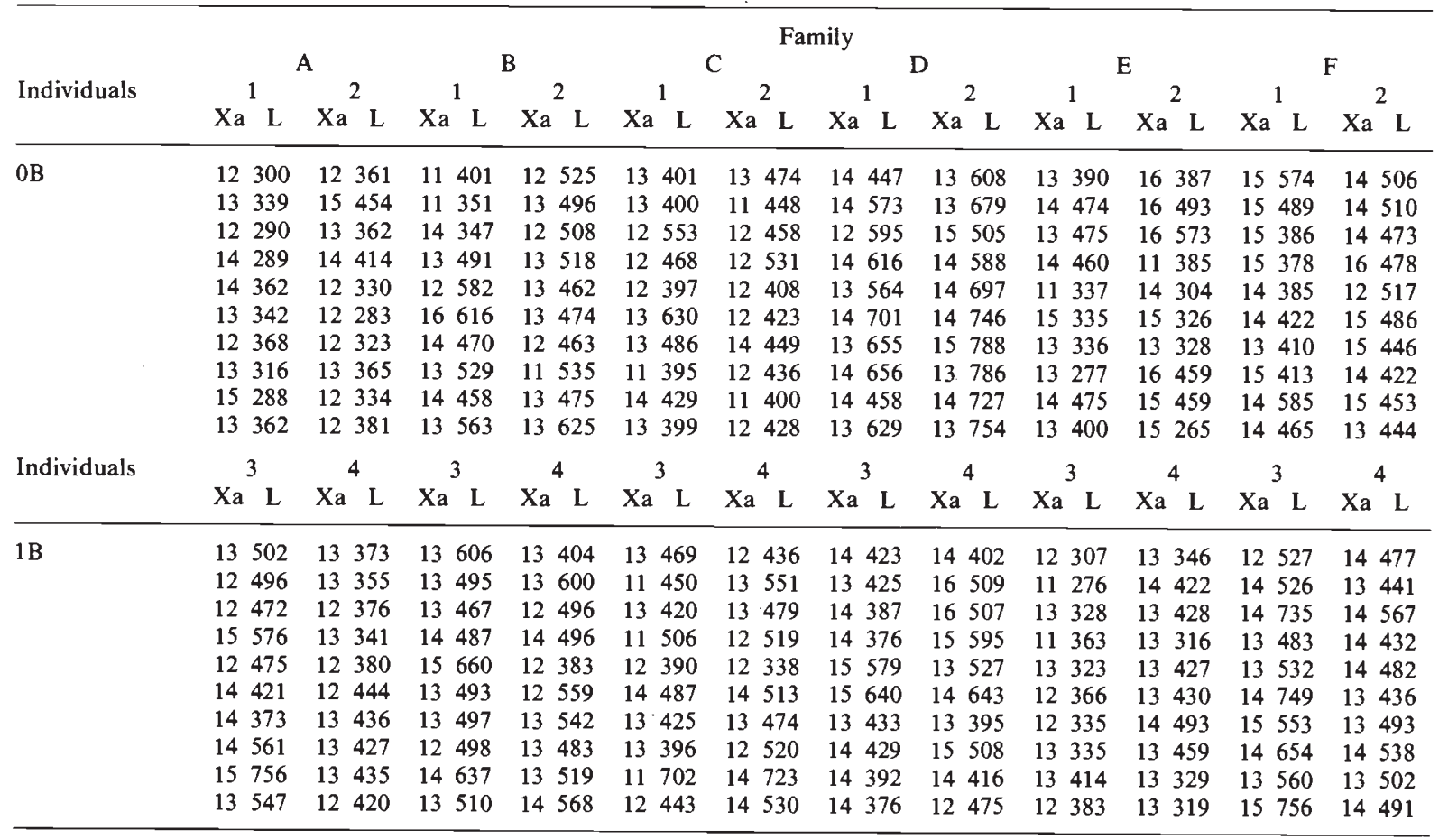


Nevertheless, the differences were small and most probably that was a random source of error and hence not affecting a particular class of individuals. In our material the mean chiasma frequency was 13.26 which implies that bivalents are usually monochiasmatic and only bivalents L1-M4 have two chiasmata.

\section{Variation in chromosome length}

Table 2 shows an analysis of variance for the total chromosome length values given in table 1 and also the final results for the different groups of chromosomes considered separately.

There are strong effects of family (F) and individual ( $\mathrm{I}$ ) and also a significant interaction between family and B chromosome (FB); the B chromosome effect is clearly non significant. From table 2 it is also evident that these effects operate at cellular level rather than specific bivalent(s) level since the results obtained from the different groups of chromosomes were practically identical to total length.

Significant effects of family and individual indicate that differences in genotypes are a primary cause of variation in chromosome length. On the other hand, the existence of a significant FB interaction suggests a differential effect of B chromosomes depending on the particular family considered. The comparison of mean chromosome length of $0 \mathrm{~B}$ and $1 \mathrm{~B}$ classes in each family, given in table 3, revealed that supernumeraries may produce a significant increase on chromosome length, a significant decrease, or even lack any effect on this parameter. The absence of an overall effect of Bs is therefore a consequence of such disparity within families.

\section{Chiasma frequency}

An analysis of variance for chiasma scores given in table 1 reveals significant effects for families and individuals (see table 5). In this case, neither the effect of B chromosomes nor their interaction with families (FB) were statistically significant.

The aim of the present study was the investigation of the variation in chromosome length as a possible mechanism for the change in chiasma frequency produced by different factors, namely, the background genotype and the presence of supernumerary chromosomes.

First of all, when pooled data are considered there is a significant dependence of the variation in chiasma frequency on chromosome length (table 4). There were no significant differences between the slopes obtained in each individual $\left(F_{23,192}=\right.$ $1 \cdot 11$ n.s.) with a mean value of $\bar{b}=3 \cdot 29 \times 10^{-3}$ $\mathrm{Xta} \times$ a.u. ${ }^{-1}$; correlation coefficients were also non significant $\left(X_{23}^{2}=25 \cdot 54\right.$ n.s. $)$. We cannot establish other comparisons for these two parameters at the remaining levels ( $\mathrm{F}, \mathrm{B}$ and $\mathrm{FB}$ ) since, as it was shown above, some of such factors have an effect not only on what we may consider the dependent variable, the chiasma frequency, but also on chromosome length and therefore different effects on both variables may give estimated values of slopes which are not representative of the true regressions.

The total number of chiasmata per cell depends almost completely on those formed in the group L1-M4; the remainder-and specially the smallest pairs-are usually monochiasmate. Nevertheless, it is worthy of mention that significant regressions were also obtained for total chiasma frequency on length of each group of autosomes.

The results of an analysis of covariance (following the method described by Steel and Torrie, 1980) considering both the classified factors and the variation in bivalent length is given in table 5. Once the effect of classified factors is eliminated, the residual variation will reflect the true regression between both variables: the slope obtained in the error row was $b=3.22 \times 10^{-3} \mathrm{Xta} \times \mathrm{a} . \mathrm{u} .^{-1}\left(s_{b}=\right.$ $\left.8.55 \times 10^{-4}, t_{\infty}=3.77, p<0.01\right)$ which is practically

Table 2 Analysis of variance for the total chromosome length of all cells analysed and also considering the different chromosome groups

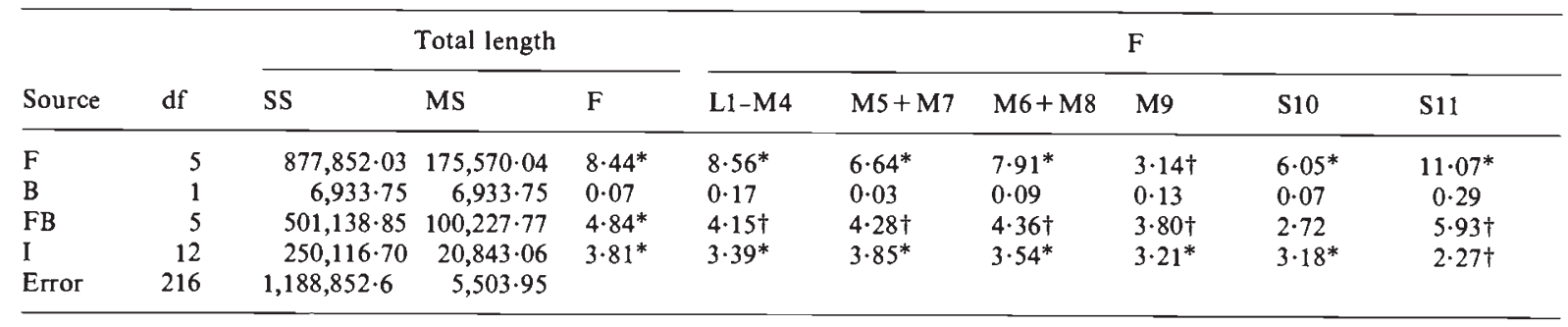

$* p<0 \cdot 01$.

$\dagger p<0.05$. 
Table 3 Mean total autosome length (true and adjusted) and mean cell chiasma scores for the individuals with 0 and $1 \mathrm{~B}$ in each family

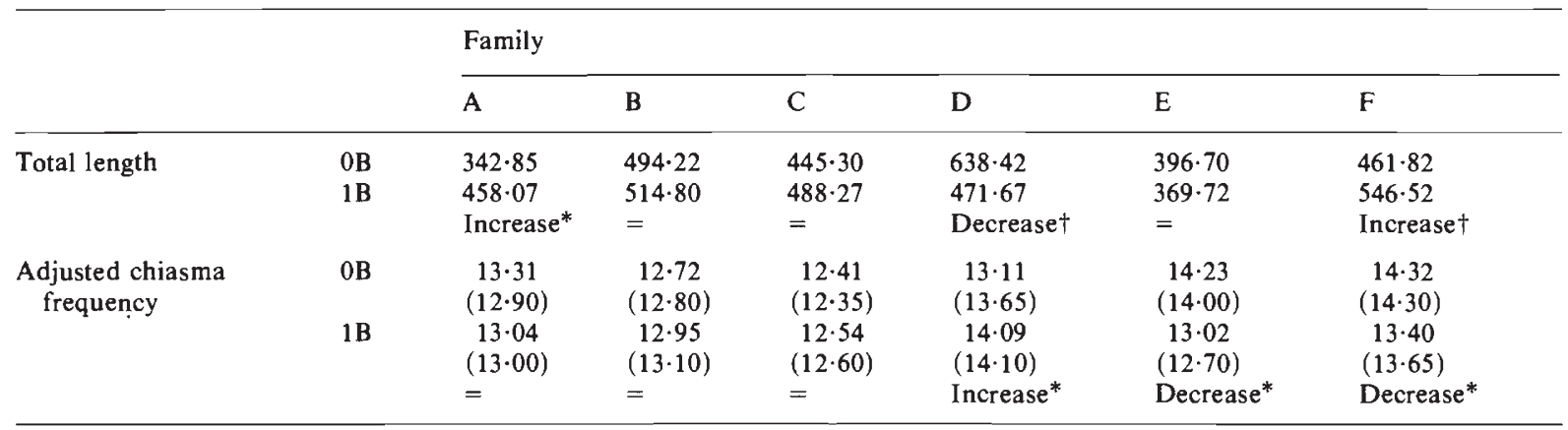

${ }^{*} p<0.01$.

$+p<0.05$.

The numbers in brackets represent the true mean cell chiasma scores.

identical to the mean between-individual slope given above. The residual variance is still quite high and therefore the degree of dependence of chiasma frequency on chromosome length is low: $r^{2}=6 \cdot 2$ per cent $(r=0 \cdot 249, p<0 \cdot 01)$. This slope can be used to adjust the observed sum of squares for each classified factor (table 5, " $y$ adjusted for $x$ "). In other words the effect of such factors is adjusted assuming that no differences of chromosome length exist. There are no drastic differences with respect to the analysis of variance using the observed data, however now, in addition to the effects of family and individual which are still significant, the interaction family-supernumerary chromosome (FB) is also significant. The comparison of adjusted mean chiasma frequencies of the individuals without Bs and those carrying one supernumerary chromosome for each family is given in table 3 . Again, the effect of B chromosomes varies in the different families and clearly the interaction between family and Bs is not parallel for the variables, chromosome length and mean chiasma frequency.

Chiasma localisation was also analysed and the results obtained showed no clear relationship with the factors considered here. The characteristics of the localisation of chiasmata in the bivalents of E. plorans will be part of a future report.

Table 4 Analysis of variance showing the dependence of the variation in chiasma frequency on chromosome length

\begin{tabular}{lrrrl}
\hline Source & \multicolumn{1}{c}{ df } & \multicolumn{1}{l}{ SS } & \multicolumn{1}{c}{ MS } & \multicolumn{1}{l}{ F } \\
\hline Chrom. length & 1 & $19 \cdot 521$ & $19 \cdot 521$ & $15 \cdot 65^{*}$ \\
Residual & 238 & $296 \cdot 342$ & 1.248 & \\
Total & 239 & 316.463 & & \\
\hline
\end{tabular}

${ }^{*} p<0.01$.

\section{DISCUSSION}

Chiasma frequency shows a wide variation both between and within different species and its dependence on the genotype is well known. There is also a great number of studies about the influence of supernumerary chromosomes on chiasma formation though this effect varies not only in magnitude but also in sign (for review, see Jones and Rees, 1982). However, the mechanism underlying such variation remains unknown. Henderson (1963) and Southern (1967) observed that bivalents were longer in cells showing higher chiasma scores and later, in a careful analysis of the meiocytes of Schistocerca gregaria, Fox (1973) confirmed such relationship and provided more details on this phenomenon which was considered an obvious candidate to account for different variations in chiasma frequency (Fox, 1973; Couzin and Fox, 1974; Riva et al., 1984).

In the grasshopper Eyprepocnemis plorans it has already been shown that the mean chiasma frequency varies notably between families and also that the effect of B chromosomes depends on the background genotype (Henriques-Gil et al., $1982 b$ ). With a more precise design this is particularly clear in the present report: there is an important effect of both the familial and the individual genotypes (table 5) and the relationship between mean chiasma frequency of males without supernumeraries and those carrying one B chromosome depends on the particular family considered (table 3).

The observed variation in chiasma frequency also depends on the variation of bivalent length (table 4). This does not seem to be a consequence of the combined effects of individual, B chromosomes and family since the removal of these factors 
Table 5 Analysis of covariance for total chiasma score

\begin{tabular}{|c|c|c|c|c|c|c|c|c|c|}
\hline \multirow[b]{2}{*}{ Source } & \multirow[b]{2}{*}{$\mathrm{df}$} & \multicolumn{3}{|c|}{ Sum of products (unadjusted $Y$ ) } & \multirow[b]{2}{*}{ MS } & \multirow[b]{2}{*}{$\mathbf{F}$} & \multicolumn{3}{|c|}{$Y$ adjusted for $X$} \\
\hline & & $\mathrm{xx}$ & $\mathrm{XY}$ & YY & & & YY & MS & $\mathrm{F}$ \\
\hline Total & 239 & $2,824,893 \cdot 93$ & 7425.95 & 316.46 & & & & & \\
\hline Family & 5 & $877,852 \cdot 03$ & $3209 \cdot 88$ & $68 \cdot 24$ & $13 \cdot 65$ & $6 \cdot 47^{*}$ & $56 \cdot 60$ & $11 \cdot 32$ & $6 \cdot 47^{*}$ \\
\hline B & 1 & $6,933 \cdot 75$ & $-91 \cdot 38$ & $1 \cdot 20$ & $1 \cdot 20$ & $0 \cdot 25$ & 1.84 & 1.84 & $0 \cdot 31$ \\
\hline $\mathrm{F} \times \mathrm{B}$ & 5 & $501,138 \cdot 85$ & $-587 \cdot 00$ & $23 \cdot 57$ & $4 \cdot 71$ & $2 \cdot 23$ & $29 \cdot 67$ & $5 \cdot 93$ & $3 \cdot 39 \dagger$ \\
\hline I & 12 & $250,116 \cdot 70$ & $1069 \cdot 75$ & $25 \cdot 35$ & $2 \cdot 11$ & $2 \cdot 29 \dagger$ & $21 \cdot 00$ & $1 \cdot 75$ & $2.03 \dagger$ \\
\hline Error & 216 & $1,188,852 \cdot 60$ & $3824 \cdot 70$ & $198 \cdot 1$ & 0.92 & & 185.80 & $0 \cdot 867$ & \\
\hline
\end{tabular}

\begin{tabular}{lll}
\hline & Slope & $r^{2}$ \\
\hline Total & $2.63 \times 10^{-3}$ & 0.061685 \\
Error & $3.22 \times 10^{-3}$ & 0.062113 \\
\hline
\end{tabular}

$* p<0.01$.

$\dagger p<0.05$.

from the total produces only small changes in the regression line with an even higher slope (table fa) 23 On another hand, if the effects of the classified factors could be explained by differences in bivalent length the values adjusted by the common slope would reveal no significant differences. In other words, under such an assumption, if bivalent length was the same in all cases we should not expect differences in chiasma frequency. Clearly, table 5 indicates that this does not occur; instead, not only the effects of family and individual are still significant at similar levels of confidence but also the analysis of variance using the adjusted values revealed a statistically significant interaction family-supernumeraries.

In Euchorthippus pulvinatus gallicus, Riva et al. (1984) observed a parallel between the effect of a supernumerary heterochromatic segment on mean cell chiasma score and the mean total bivalent length, suggesting the length effect as a mechanism for the action of such segment. Their results, however, also indicate that the variation in autosome length at diplotene was not responsible for the total bulk of increase in chiasma frequency, attributing to the variation in the genetic background a possible role for such lack of a complete correspondence. In the case of Eyprepocnemis plorans we provide here direct evidence that the genetic background (at a familial or individual level) and also the supernumerary chromosome interacting with the genotypes have an effect on bivalent length, as shown in table 2 . This supports the assumption of Riva et al. (1984) in that all effects on length operate at cellular level rather than specific bivalent level given the strict parallel between the length of all chromosome groups including those invariably monochiasmatic.
Nevertheless as it was already shown, the elimination of genotypic effects does not improve the regression of chiasma frequency on bivalent length. Additionally we found no relationships between the effects of B chromosomes within each family on either bivalent length or chiasma frequency (table 3 ). In consequence, our data do not support the variation in autosomal length at first meiotic prophase as the mechanism for the effects of genotypes and B chromosomes on chiasma frequency in the grasshopper E. plorans.

This does not necessarily imply that the effects of such factors on bivalent length and chiasma frequency are always independent. It is important to note that in our material mean chiasma frequencies-varying from $12 \cdot 2$ to $14 \cdot 7$-are quite low (11 chiasmata, one per bivalent, may be considered the minimum in a cell with normal pairing). So there is a possibility that in other materials, from this or another species, with higher chiasma frequencies (like the case of Schistocerca gregaria studied by Fox, 1973) a different and wider variation in genotypes affecting this parameter exists and a consistent relationship between the effects on chiasma frequency and bivalent length may be found.

It could be also argued that supernumerary segments and B chromosomes, in different species or genetic backgrounds, may have different mechanisms of affecting chiasma frequency. We think, however, that it is extremely unlikely that for all cases of effects of supernumerary segments or chromosomes on chiasma formation reported in the literature there is a different underlying mechanism. The existence of a common mechanism at least in part seems mure likely. Though there is a positive correlation between chiasma 
frequency and bivalent length, our results indicate that this relationship is not responsible for the effects of genotypes and B chromosomes on chiasma formation.

Acknowledgement This work was partially supported by a grant of the Comisión Asesora de Investigación Científica y Técnica de España.

\section{REFERENCES}

COUZIN, D. A. AND FOX, D. P. 1974. Variation in chiasma frequency during tulip anther development. Chromosoma, 46, 173-179.

DEARN, J. M. 1975. Chiasma frequency and adaptability in locust. Evolution, 29, 572-574.

FOX, D. P. 1973. The control of chiasma distribution in the locust Schistocerca gregaria (Forskal). Chromosoma, 43, 289-328.

HENDERSON, S. A. 1963. Chiasma distribution at diplotene in a locust (Schistocerca gregaria) Heredity, 18, 173-190.

HENRIQUES-GIL, N., SANTOS, J. L. AND GIRALDEZ, R. 1982a. B-chromosome polymorphism and interchromosomal chiasma interference in Eyprepocnemis plorans (Acrididae, Orthoptera). Chromosoma, 85, 349-359.

HENRIQUES-GIL, N., SANTOS, J. L. AND GIRALDEZ, R. $1982 \mathrm{~b}$. Genotype-dependent effect of B-chromosomes and chiasma frequency in Eyprepocnemis plorans (Acrididae, Orthoptera). Genetica, 59, 223-227.
HENRIQUES-GIL, N., SANTOS, J. L. AND ARANA, P. 1984. Evolution of a complex B-chromosome polymorphism in the grasshopper Eyprepocnemis plorans. Chromosoma, 89, 290293.

JONES, R. N. AND REES, H. 1982. B-chromosomes. Academic Press, New York.

MAYNARD SMITH, J. 1977. Why the genome does not congeal? Nature, 268, 693-696.

NOLTE, D. J. 1968. The chiasma-inducing pheromone of locusts. Chromosoma, 23, 346-358.

NOLTE, D. J., DÉSI, I. AND MEYERS, B. 1969. Genetic and environmental factors affecting chiasma formation in locusts. Chromosoma, 27, 145-155.

REES, H. AND AHMAD, K. 1963. Chiasma frequencies in Lolium populations. Evolution, 17, 575-579.

REES, H. AND DALE, P. J. 1974. Chiasmata and variability in Lolium and Festuca populations. Chromosoma, 47, 335351.

RIVA, E., FOX, D. P., GIRALDEZ, R. AND SANTOS, J. L. 1984. Chiasma frequency and distribution in the presence and absence of supernumerary chromosome segments in the grasshopper Euchorthippus pulvinatus gallicus. Heredity, 53, 101-106.

SHAW, D. D. 1971. Genetic and environmental components of chiasma control. I. Spatial and temporal variation in Schistocerca gregaria. Chromosoma, 34, 281-301.

SHAW, D. D. 1972. Genetic and environmental components of chiasma control. II. The response to selection in Schistocerca. Chromosoma, 37, 297-308.

SOUTHERN, D. I. 1967. Chiasma distribution in Truxaline grasshoppers. Chromosoma, 22, 164-191.

STEEL, R. G. D. AND TORRIE, J. H. 1980. Principles and procedures of statistic. A biometrical approach. McGraw-Hill Book Company. 